\title{
Socio-economic and demographic variations in school lunch participation of French children aged 3-17 years
}

\author{
Carine Dubuisson ${ }^{1, *}$, Sandrine Lioret ${ }^{1}$, Ariane Dufour ${ }^{1}$, Gloria Calamassi-Tran ${ }^{1}$, \\ Jean-Luc Volatier ${ }^{2}$, Lionel Lafay ${ }^{1}$ and Dominique Turck ${ }^{3}$ \\ 'French Agency for Food, Environmental and Occupational Health Safety (ANSES), Direction of Risk Assessment \\ for Nutrition and Food Safety, Dietary Survey Unit - Nutritional Epidemiology, 27-31 Avenue du Général \\ Leclerc, 94701 Maisons Alfort Cedex, France: ${ }^{2}$ French Agency for Food, Environmental and Occupational \\ Health Safety (ANSES), Office of Scientific Support for Risk Assessment, Maisons-Alfort, France: ${ }^{3}$ Department of \\ Paediatrics, Jeanne de Flandre Children's Hospital and Lille University Faculty of Medicine, Lille, France
}

Submitted 18 December 2009: Accepted 21 June 2010: First published online 12 August 2010

\begin{abstract}
Objective: To assess school canteen attendance in a French nationally representative sample of children and to analyse its association with the socioeconomic and demographic characteristics of the children and their families.

Design: Data from the second French national cross-sectional food consumption survey (INCA2), performed in 2006-2007, were used. Information on usual weekly school canteen attendance was collected through a self-reported questionnaire, and demographic and socio-economic variables through a face-to-face questionnaire. The associations between school canteen attendance and the socio-economic and demographic variables were investigated by multivariate logistic regression analyses.

Setting: The INCA2 sample was representative of the children aged 3-17 years in France.

Subject: Analysis was performed on 1413 schoolchildren who completed the school canteen attendance questions.

Results: Some $65 \cdot 6 \%$ of schoolchildren aged 3-17 years had school lunch at least once weekly. This rate of attendance was positively correlated with age. Whatever the school level, school canteen attendance was positively associated with the educational level of the caregiver/parent. In pre- and elementary-school children, enrolment at the school canteen was also higher when the caregiver/parent worked, or in single-parent families. In secondary-school children, school lunch participation decreased with children living in more densely populated areas and increased with the level of the household's living standards.

Conclusions: School canteen attendance was positively associated with children's socio-economic background. This could reduce the effectiveness of the forthcoming school meal composition regulations designed to improve the diet of children from deprived backgrounds, who are more likely to have unhealthy food habits.
\end{abstract}

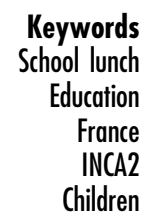

To promote healthier eating behaviours and to tackle the increasing prevalence of overweight and obesity in the paediatric population, many industrialised countries have set up school nutrition policies ${ }^{(1)}$. Controlling the availability of food items on school premises is one way to lead children and adolescents towards healthier food choices at lunchtime ${ }^{(1-5)}$ and may help to balance their energy intake ${ }^{(5)}$. In France, the school food environment has been included in national nutrition policies since the implementation of the first National Nutrition and Health Plan (PNNS1) ${ }^{(6)}$ in 2001. The morning snack was discouraged in elementary schools in 2004 by a circulart from the Ministry of Education ${ }^{(7)}$, whereas beverages and food vending machines have been banned by law $^{(8)}$ from school premises since 2005. In 2006, the second National Nutrition and Health Plan (PNNS2) ${ }^{(6)}$ proposed several actions dedicated to schools, among which was improving the nutritional quality of food offered. Indeed, balanced school meals must be provided in order to

$\dagger$ In France, a circular is a text issued by a ministry intended to be applied by public officers. It is not compulsory but acts as a recommendation. 
encourage schoolchildren to adopt healthier dietary patterns. In France, new national and compulsory regulations will define standards for school meal composition and portion size and will replace the national recommendations introduced on 25 June 2001 by an interministerial circular ${ }^{(9,10)}$.

From a public health point of view, the impact of this regulation to improve the dietary habits of schoolchildren will depend first on the school canteen attendance rate and second on whether or not the children with unhealthy eating behaviours actually attend school canteens. Nearly six million French children aged 3-17 years took lunch at school, almost five days per week, in the late $1990 \mathrm{~s}^{(11)}$. The rate of school canteen attendance (at least three school lunches per week) in lower and upper secondary French schools was estimated at $67 \%$, based on a national school survey conducted in 2005-2006 ${ }^{(9)}$. Previous studies performed in 1998-1999 $9^{(12,13)}$ showed that this attendance was strongly associated with socio-economic and demographic factors. Many of these factors are at the same time associated with dietary patterns, as well as with childhood overweight and obesity in France ${ }^{(14,15)}$. An analysis of the socioeconomic factors involved in school canteen attendance could consequently be useful to identify whether social and economic measures are needed to accompany school food environment policies. Given this background, the objectives of the present study were to: (i) estimate the attendance at school canteens in 2006-2007, based on a nationally representative sample of French children; and (ii) analyse the associations between school meal attendance and the socioeconomic and demographic characteristics of the children.

\section{Materials and methods}

Data from the second French national cross-sectional food consumption survey, INCA2 (2006-2007), were used in the analysis.

\section{Participants}

The French INCA2 food consumption survey was carried out between December 2005 and May 2007 by the French Food Safety Agency (AFSSA). A complex sampling design, which has been described elsewhere ${ }^{(16-18)}$, was used to obtain a nationally representative sample of French people. Briefly, two independent random samples of 3- to 17-yearold children and 18- to 79-year-old adults were drawn on the basis of a multistage cluster sampling technique. The sampling frame was extracted from the national census published by INSEE (French National Institute of Statistics and Economic Studies). First, 181 primary geographical units, stratified by region of residence and size of urban area, were randomly selected with probability proportional to size. Then, households were randomly drawn from each primary unit and two independent sampling frames were set up: the first restricted to households including at least one child and the second including households with or without children. Finally, within each household, either a child or an adult was randomly selected. A participation rate of $69 \%$ was obtained for children, yielding a sample of 1455 children aged 3-17 years.

\section{Measurements}

Diet was assessed using an open-ended $7 \mathrm{~d}$ food record. Each day of the diary was divided into three main meals and three between-meals. For the main meals, the consumption location was chosen from six proposals (at home, at a canteen, at a friend's home, at a fast-food outlet, at a restaurant, other). Behavioural, demographic and socio-economic variables were collected by selfreported and face-to-face questionnaires. A trained and certified investigator delivered the $7 \mathrm{~d}$ record and the selfadministered questionnaire to the home and explained to the parents and their child how to complete them. Children aged 10 years or less were helped by their parents or caregivers to fill out the documents. After the survey week, the investigator came back and checked the accuracy of the information reported in the documents. The face-to-face questionnaire was then administered partly to the child and partly to his/her adult caregiver (mainly the mother; 80\%).

The self-reported questionnaire included questions on usual weekly school canteen attendance and, in cases where the child never attended the school canteen, on the reasons for not taking lunch at school. Several answers were proposed; otherwise a free answer could be noted. The face-to-face questionnaire included questions on socio-economic issues (household's income, head of household's and child caregiver's occupations, employment status and educational level), as well as indices of household living standards such as having gone away on holiday for more than 4 days within the last 12 months' (yes/no), 'number of cars in the household', 'number of domestic electrical appliances', 'how the financial situation was perceived' (positively/negatively), 'financial access to desired food products' (yes/no), 'whether the idea of lacking food would be a concern' (yes/no), 'giving up health care for financial reasons' (yes/no) and 'housing occupation status' (first-time buyer or owner/tenant/tenant in social housing and others (free housing, etc)). Other information, such as region, type of settlement in which the household was located, household composition and child's school level, was collected during the face-to-face interview.

The survey was approved by the Data Protection Authority (Commission Nationale Informatique et Libertés, CNIL).

\section{Data analysis}

All analyses were computed with the STATA statistical software package release 10 (StataCorp LP, College Station, TX, USA). Individual data were weighted for unequal 
sampling probabilities and for differential non-responses by region, urban area size, age, gender, head of the household's occupation, size of the household and season. The external data used came from the INSEE national data set for 2005.

Children aged 3-17 years were classified according to the school level that they attended (pre-school, elementary, lower and upper secondary schools). Twentysix unschooled children were excluded and sixteen schoolchildren did not complete the questions on school canteen attendance. Analysis was carried out on 1413 children.

Usual school canteen attendance was defined with a three-class variable: 'regular users' (at least 3 lunches/ week), 'occasional users' (1 or 2 lunches/week) and 'nonusers' (less than 1 lunch/week). Another two-class variable was also created to be used in logistic regression ('regular or occasional users' $v$. 'non-users').

The occupational status of the child's caregiver was classified as 'high' (executives, top management and professional categories), 'medium' (employees, technicians and similar), 'low' (manual workers, unemployed) or 'economically inactive' (retired and non-working mothers or fathers). An indicator variable on his/her employment status was also created: 'working' or 'nonworking' (retired, non-working mother or father, unemployed). The educational level of the child's caregiver was defined according to three levels: 'high', 'middle' or 'low'. 'High' was assigned to university level, 'middle' to secondary school level and 'low' to elementary school level. An additional socio-economic factor, called the 'household living standards index', was used. It was derived from correspondence analysis performed on the matrix based on the variables coding for the household's living standards, as previously described ${ }^{(19)}$. The score of each household on the first principal component was used as the summary index, and divided into tertiles. This index did not include the household's income because of $21 \%$ of missing values, but they were compared with each other for external validation.

The household composition was described using two variables: the number of children (one; two; three or more) and the number of adults (one; two or more).

The prevalence of school canteen attendance was estimated by school level and for each socio-economic factor, taking into account the complex design of the survey (svy: procedures). Pearson $\chi^{2}$ tests were used to compare frequencies. Critical $P$ value was set at $P=0 \cdot 05$.

Multivariate logistic regression analyses were performed to investigate the associations between school canteen attendance as the dependent variable and the socio-economic (child caregiver's occupational and employment status, educational level, household wealth index) and demographic (child's age and gender, region, urban area size, child caregiver's age and household composition) variables (svy: logistic procedure). All of these variables were first introduced simultaneously and then removed step-by-step from the model (according to the $P$ value) to avoid collinearity. Critical $P$ values that selected the final remaining variables in the model were set at $P=0 \cdot 05$. The lowest category of each socioeconomic indicator was taken as the reference group to estimate the odds ratios.

\section{Results}

\section{Description of the participants}

The general characteristics of the participants are presented in Table 1 according to their school level. Four specific characteristics for each school level were observed. First, the average age of the caregiver/parent increased with school level. Second, caregivers/parents of pre-school children had higher educational levels than those of children attending upper secondary school. Third, 35\% of the younger children (pre-school and elementary school) had at least two sisters or brothers, whereas almost half of the children in upper secondary schools were alone in the household. Fourth, children attending upper secondary schools lived more often in city centres than in rural areas (scattered settlements or villages) and young children lived more frequently in suburban areas. The other socio-economic (occupational status, employment status, household wealth index) and demographic (number of adults in the household, gender ratio) variables were equally divided into the four school level categories.

\section{School canteen attendance}

Nearly two-thirds of French children had lunch at school at least once weekly (Table 1). The highest attendance was observed in lower secondary school (71.5\%) and the lowest in pre-school $(59 \cdot 0 \% ; P=0.004)$. Most children attending the school canteen used it regularly. The occasional user rate was higher among young schoolchildren $(10 \cdot 4 \%$ and $14.6 \%$ in pre-school and elementary school, respectively).

According to bivariate analysis, school lunch attendance at least once weekly did not differ significantly between girls and boys. However, school canteen attendance varied with other socio-economic and demographic factors (Tables 2 and 3). At every school level, school canteen attendance rose with the educational level and occupational status of the child's caregiver. Their working status also had a role, mainly for young schoolchildren. When one of the parents did not work, the child often did not have lunch at school. Conversely, young children from single-parent families, or whose caregiver was older, had lunch at school more frequently. The school canteen attendance of older schoolchildren also rose in line with the living standards index of the household, with those from deprived households attending 
Table 1 Characteristics of 3- to 17-year-old children sampled in the second French national cross-sectional food consumption survey (INCA2, 2006-2007), according to school level

\begin{tabular}{|c|c|c|c|c|c|c|c|c|c|c|c|}
\hline \multirow{3}{*}{$n_{\text {raw }} / n_{\text {weighted }}$} & \multicolumn{2}{|c|}{ Pre-school } & \multicolumn{2}{|c|}{ Elementary school } & \multicolumn{2}{|c|}{ Lower secondary school } & \multicolumn{2}{|c|}{ Upper secondary school } & \multicolumn{2}{|c|}{ All 3-17 years } & \multirow[t]{2}{*}{$P$ value } \\
\hline & \multicolumn{2}{|c|}{$192 / 257$} & \multicolumn{2}{|c|}{$416 / 537$} & \multicolumn{2}{|c|}{$511 / 405$} & \multicolumn{2}{|c|}{$294 / 215$} & \multicolumn{2}{|c|}{$1413 / 1414$} & \\
\hline & $\%$ or Mean & $95 \% \mathrm{Cl}$ or $\mathrm{SE}$ & $\%$ or Mean & $95 \% \mathrm{Cl}$ or SE & $\%$ or Mean & $95 \% \mathrm{Cl}$ or $\mathrm{SE}$ & $\%$ or Mean & $95 \% \mathrm{Cl}$ or SE & $\%$ or Mean & $95 \% \mathrm{Cl}$ or SE & \\
\hline Gender male $(\%, 95 \% \mathrm{Cl})$ & $51 \cdot 9$ & $44 \cdot 0,59 \cdot 8$ & $53 \cdot 8$ & $48 \cdot 4,59 \cdot 3$ & $48 \cdot 7$ & $43 \cdot 1,54 \cdot 4$ & $46 \cdot 7$ & $38 \cdot 1,55 \cdot 3$ & $50 \cdot 9$ & $47 \cdot 6,54 \cdot 3$ & 0.43 \\
\hline Age (years; mean, SE) & $4 \cdot 8$ & 0.97 & $8 \cdot 5$ & $1 \cdot 56$ & $13 \cdot 2$ & $1 \cdot 55$ & $16 \cdot 2$ & $0 \cdot 88$ & $10 \cdot 4$ & $4 \cdot 06$ & $<0.001$ \\
\hline School canteen attendance $(\%, 95 \% \mathrm{Cl})$ & & & & & & & & & & & $<0.001$ \\
\hline Regular user ( $\geq 3 /$ week) & $48 \cdot 6$ & $40 \cdot 7,56 \cdot 4$ & $50 \cdot 3$ & $44 \cdot 7,56 \cdot 0$ & $67 \cdot 1$ & $62 \cdot 4,71 \cdot 7$ & $59 \cdot 0$ & $49 \cdot 4,68 \cdot 6$ & $56 \cdot 1$ & $52 \cdot 8,59 \cdot 4$ & \\
\hline Occasional user (1-2/week) & $10 \cdot 4$ & $5 \cdot 4,15 \cdot 4$ & $14 \cdot 6$ & $10 \cdot 6,18 \cdot 5$ & $4 \cdot 4$ & $2 \cdot 5,6 \cdot 4$ & $5 \cdot 4$ & $2 \cdot 4,8 \cdot 3$ & $9 \cdot 5$ & $7 \cdot 6,11 \cdot 5$ & \\
\hline Non-user (<1/week) & $41 \cdot 0$ & $33 \cdot 3,48 \cdot 8$ & $35 \cdot 1$ & $29 \cdot 6,40 \cdot 5$ & $28 \cdot 5$ & $23 \cdot 9,33 \cdot 1$ & $35 \cdot 6$ & $25 \cdot 5,45 \cdot 7$ & $34 \cdot 4$ & $31 \cdot 1,37 \cdot 6$ & \\
\hline CC's age (years; mean, SE) & & & & & & & & & & & $<0.001$ \\
\hline$n$ & 189 & & 411 & & 500 & & 291 & & 1391 & & \\
\hline Age & $35 \cdot 5$ & 0.50 & $39 \cdot 6$ & 0.48 & $44 \cdot 0$ & 0.47 & $46 \cdot 8$ & 0.52 & $41 \cdot 2$ & $0 \cdot 29$ & \\
\hline CC's occupational status $(\%, 95 \% \mathrm{Cl})$ & & & & & & & & & & & $0 \cdot 15$ \\
\hline 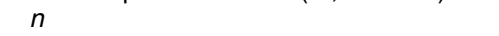 & 190 & & 411 & & 500 & & 284 & & 1385 & & \\
\hline High & $8 \cdot 7$ & $4 \cdot 4,12 \cdot 9$ & $8 \cdot 9$ & $5 \cdot 7,12 \cdot 2$ & $9 \cdot 4$ & $6 \cdot 4,12 \cdot 3$ & $15 \cdot 6$ & $10 \cdot 6,20 \cdot 6$ & $10 \cdot 0$ & $8 \cdot 1,11 \cdot 9$ & \\
\hline Medium & $46 \cdot 3$ & $38 \cdot 2,54 \cdot 3$ & $55 \cdot 7$ & $50 \cdot 3,61 \cdot 0$ & $55 \cdot 4$ & $49 \cdot 9,60 \cdot 9$ & $52 \cdot 0$ & $42 \cdot 9,61 \cdot 1$ & $53 \cdot 3$ & $50 \cdot 0,56 \cdot 7$ & \\
\hline Low (including unemployed) & $18 \cdot 7$ & $12 \cdot 1,25 \cdot 3$ & $15 \cdot 8$ & $11 \cdot 8,19 \cdot 9$ & $14 \cdot 4$ & $10 \cdot 5,18 \cdot 3$ & $9 \cdot 3$ & $5 \cdot 4,13 \cdot 2$ & $15 \cdot 0$ & $12 \cdot 4,17 \cdot 5$ & \\
\hline Economically inactive & $26 \cdot 4$ & $19 \cdot 2,33 \cdot 5$ & $19 \cdot 6$ & $14 \cdot 7,24 \cdot 5$ & $20 \cdot 8$ & $15 \cdot 7,25 \cdot 9$ & $23 \cdot 0$ & $12 \cdot 0,34 \cdot 0$ & $21 \cdot 7$ & $18 \cdot 6,24 \cdot 8$ & \\
\hline CC's employment status $(\%, 95 \% \mathrm{Cl})$ & & & & & & & & & & & 0.29 \\
\hline$n$ & 192 & & 416 & & 510 & & 294 & & 1412 & & \\
\hline Working & $64 \cdot 7$ & $56 \cdot 6,72 \cdot 8$ & $73 \cdot 2$ & $68 \cdot 1,78 \cdot 3$ & $73 \cdot 9$ & $68 \cdot 7,79 \cdot 1$ & $71 \cdot 7$ & $61 \cdot 3,82 \cdot 0$ & $71 \cdot 6$ & $68 \cdot 5,74 \cdot 8$ & \\
\hline Non-working & $35 \cdot 3$ & $27 \cdot 2,43 \cdot 4$ & $26 \cdot 8$ & $21 \cdot 7,31 \cdot 9$ & $26 \cdot 1$ & $20 \cdot 9,31 \cdot 3$ & $28 \cdot 3$ & $18 \cdot 0,38 \cdot 7$ & $28 \cdot 4$ & $25 \cdot 2,31 \cdot 5$ & \\
\hline CC's educational level $(\%, 95 \% \mathrm{Cl})$ & & & & & & & & & & & 0.009 \\
\hline$n$ & 192 & & 413 & & 507 & & 293 & & 1405 & & \\
\hline High & $42 \cdot 1$ & $34 \cdot 3,49 \cdot 9$ & $36 \cdot 4$ & $31 \cdot 2,41 \cdot 6$ & $29 \cdot 6$ & $24 \cdot 6,34 \cdot 7$ & $27 \cdot 5$ & $20 \cdot 9,34 \cdot 1$ & $34 \cdot 1$ & $31 \cdot 2,37 \cdot 1$ & \\
\hline Medium & $46 \cdot 3$ & $38 \cdot 4,54 \cdot 2$ & $53 \cdot 5$ & $48 \cdot 0,59 \cdot 0$ & $53 \cdot 1$ & $47 \cdot 9,58 \cdot 3$ & $50 \cdot 6$ & $41 \cdot 9,59 \cdot 3$ & $51 \cdot 7$ & $48 \cdot 4,54 \cdot 9$ & \\
\hline Low & $11 \cdot 6$ & $6 \cdot 5,16 \cdot 6$ & $10 \cdot 1$ & $6 \cdot 8,13 \cdot 4$ & $17 \cdot 3$ & $12 \cdot 7,21 \cdot 8$ & $21 \cdot 9$ & $11 \cdot 3,32 \cdot 5$ & $14 \cdot 2$ & $11 \cdot 5,16 \cdot 9$ & \\
\hline No. of children in household $(\%, 95 \% \mathrm{Cl})$ & & & & & & & & & & & $<0.001$ \\
\hline n & 192 & & 416 & & 511 & & 294 & & 1413 & & \\
\hline 1 child & $11 \cdot 9$ & $8 \cdot 0,15 \cdot 8$ & $12 \cdot 2$ & $9 \cdot 1,15 \cdot 2$ & $30 \cdot 5$ & $25 \cdot 8,35 \cdot 2$ & $44 \cdot 3$ & $36 \cdot 3,52 \cdot 2$ & $22 \cdot 3$ & $20 \cdot 0,24 \cdot 5$ & \\
\hline 2 children & $52 \cdot 6$ & $45 \cdot 0,60 \cdot 2$ & $53 \cdot 6$ & $47 \cdot 9,59 \cdot 3$ & $44 \cdot 2$ & $38 \cdot 7,49 \cdot 8$ & $33 \cdot 0$ & $25 \cdot 5,40 \cdot 4$ & $47 \cdot 6$ & $44 \cdot 5,50 \cdot 7$ & \\
\hline 3 or more children & $35 \cdot 5$ & $27 \cdot 5,43 \cdot 4$ & $34 \cdot 3$ & $28 \cdot 5,40 \cdot 0$ & $25 \cdot 3$ & $20 \cdot 6,29 \cdot 9$ & $22 \cdot 8$ & $12 \cdot 3,33 \cdot 3$ & $30 \cdot 2$ & $26 \cdot 9,33 \cdot 4$ & \\
\hline No. of adults in household $(\%, 95 \% \mathrm{Cl})$ & & & & & & & & & & & $0 \cdot 14$ \\
\hline$n$ & 192 & & 416 & & 511 & & 294 & & 1413 & & \\
\hline 1 adult (single-parent family) & $7 \cdot 1$ & $3 \cdot 1,11 \cdot 1$ & $9 \cdot 2$ & $6 \cdot 2,12 \cdot 2$ & $12 \cdot 1$ & $8 \cdot 6,15 \cdot 6$ & $13 \cdot 6$ & $8 \cdot 9,18 \cdot 3$ & $10 \cdot 3$ & $8 \cdot 5,12 \cdot 2$ & \\
\hline 2 or more adults & $92 \cdot 9$ & $88.9,96 \cdot 9$ & $90 \cdot 8$ & $87 \cdot 8,93 \cdot 8$ & $87 \cdot 9$ & $84 \cdot 4,91 \cdot 4$ & $86 \cdot 4$ & $81 \cdot 7,91 \cdot 1$ & $89 \cdot 7$ & $87 \cdot 8,91 \cdot 5$ & \\
\hline Settlement $(\%, 95 \% \mathrm{Cl})$ & & & & & & & & & & & 0.003 \\
\hline$n$ & 192 & & 416 & & 511 & & 294 & & 1413 & & \\
\hline Scattered & $14 \cdot 0$ & $8 \cdot 9,19 \cdot 1$ & $11 \cdot 2$ & $7 \cdot 3,15 \cdot 2$ & $13 \cdot 6$ & $9 \cdot 5,17 \cdot 8$ & $8 \cdot 4$ & $5 \cdot 0,11 \cdot 8$ & $12 \cdot 0$ & $9 \cdot 5,14 \cdot 5$ & \\
\hline Village & $19 \cdot 6$ & $13 \cdot 3,25 \cdot 8$ & $33 \cdot 7$ & $27 \cdot 7,39 \cdot 6$ & $31 \cdot 9$ & $26 \cdot 5,37 \cdot 2$ & $21 \cdot 7$ & $16 \cdot 1,27 \cdot 4$ & $28 \cdot 8$ & $25 \cdot 3,32 \cdot 3$ & \\
\hline Suburban & $44 \cdot 7$ & $37 \cdot 1,52 \cdot 3$ & $30 \cdot 7$ & $25 \cdot 3,36 \cdot 1$ & $32 \cdot 3$ & $27 \cdot 0,37 \cdot 7$ & $40 \cdot 9$ & $32 \cdot 4,49 \cdot 4$ & $35 \cdot 3$ & $31 \cdot 8,38 \cdot 8$ & \\
\hline City centre & $21 \cdot 7$ & $15 \cdot 2,28 \cdot 2$ & $24 \cdot 4$ & $19 \cdot 9,29 \cdot 0$ & $22 \cdot 2$ & $17 \cdot 4,26 \cdot 9$ & $28 \cdot 9$ & $19 \cdot 7,38 \cdot 1$ & $24 \cdot 0$ & $21 \cdot 0,26 \cdot 9$ & \\
\hline Household wealth index $(\%, 95 \% \mathrm{Cl})$ & & & & & & & & & & & $0 \cdot 15$ \\
\hline$n$ & 192 & & 416 & & 511 & & 294 & & 1413 & & \\
\hline Low & $41 \cdot 2$ & $33 \cdot 2,49 \cdot 2$ & $32 \cdot 7$ & $27 \cdot 4,37 \cdot 9$ & $33 \cdot 6$ & $28 \cdot 5,38 \cdot 7$ & $34 \cdot 4$ & $24 \cdot 2,44 \cdot 6$ & $34 \cdot 8$ & $31 \cdot 4,38 \cdot 1$ & \\
\hline Medium & $36 \cdot 3$ & $29 \cdot 0,43 \cdot 6$ & $32 \cdot 6$ & $27 \cdot 2,37 \cdot 9$ & $31 \cdot 2$ & $26 \cdot 4,36 \cdot 1$ & $32 \cdot 3$ & $24 \cdot 7,39 \cdot 9$ & $32 \cdot 8$ & $29 \cdot 8,35 \cdot 9$ & \\
\hline High & 22.5 & $15 \cdot 9,29 \cdot 0$ & $34 \cdot 8$ & $29 \cdot 8,39 \cdot 8$ & $35 \cdot 2$ & $30 \cdot 3,40 \cdot 0$ & $33 \cdot 3$ & $26 \cdot 2,40 \cdot 4$ & $32 \cdot 4$ & $29 \cdot 5,35 \cdot 3$ & \\
\hline
\end{tabular}


Table 2 Prevalence of regular and occasional school canteen (SC) attendance (\%, 95\% Cl), and multivariate-adjusted odds ratios (ORa, $95 \% \mathrm{Cl}$ ) for SC attendance according to socioeconomic factors among sampled children attending pre-schools and elementary schools in the second French national cross-sectional food consumption survey (INCA2, 2006-2007)

\begin{tabular}{|c|c|c|c|c|c|c|c|c|c|c|c|c|}
\hline & \multicolumn{4}{|c|}{ Pre-school } & \multicolumn{4}{|c|}{ Elementary school } & \multicolumn{4}{|c|}{ Total } \\
\hline & \multicolumn{2}{|c|}{ SC attendance } & \multirow{2}{*}{$\begin{array}{l}\mathrm{ORa}^{*} \\
(n \text { 192) }\end{array}$} & \multirow[b]{2}{*}{$95 \% \mathrm{Cl}$} & \multicolumn{2}{|c|}{ SC attendance } & \multirow{2}{*}{$\begin{array}{l}\mathrm{ORa}^{*} \\
(n 413)\end{array}$} & \multirow[b]{2}{*}{$95 \% \mathrm{Cl}$} & \multicolumn{2}{|c|}{ SC attendance } & \multirow{2}{*}{$\begin{array}{l}\mathrm{ORa}^{*} \\
(n 597)\end{array}$} & \multirow[b]{2}{*}{$95 \% \mathrm{Cl}$} \\
\hline & $\%$ & $95 \% \mathrm{Cl}$ & & & $\%$ & $95 \% \mathrm{Cl}$ & & & $\%$ & $95 \% \mathrm{Cl}$ & & \\
\hline \multicolumn{13}{|l|}{ Gender } \\
\hline Boys & 63 & 52,74 & & & 67 & 60,74 & & & 66 & 60,72 & & \\
\hline Girls & 55 & 44,65 & & & 63 & 55,71 & & & 60 & 54,66 & & \\
\hline$P$ value & \multicolumn{2}{|c|}{$0 \cdot 27$} & & & \multicolumn{2}{|c|}{0.43} & \multicolumn{6}{|c|}{$0 \cdot 18$} \\
\hline $\begin{array}{l}\text { Child's age, OR }(95 \% \mathrm{Cl}) \\
\quad P \text { value }\end{array}$ & \multicolumn{2}{|c|}{0.34} & & & \multicolumn{2}{|c|}{$\begin{array}{l}0 \cdot 84,1 \cdot 13 \\
0 \cdot 72\end{array}$} & \multicolumn{4}{|c|}{$\begin{array}{l}0 \cdot 96,1 \cdot 13 \\
0.29\end{array}$} & & \\
\hline \multicolumn{13}{|l|}{ Region } \\
\hline lle de France & 84 & 72,95 & 0.68 & $0 \cdot 14,3 \cdot 16$ & 79 & 67,91 & $1 \cdot 17$ & $0.39,3.53$ & 81 & 72,89 & 0.78 & $0.31,1.97$ \\
\hline North-west & 47 & 29,65 & $0 \cdot 11$ & $0.03,0.43$ & 79 & 71,88 & $1 \cdot 32$ & $0.50,3.53$ & 69 & 61,77 & 0.52 & $0 \cdot 23,1 \cdot 16$ \\
\hline North-east & 47 & 30,63 & $0 \cdot 12$ & $0.03,0.46$ & 38 & 27,48 & $0 \cdot 16$ & $0.06,0.42$ & 41 & 31,50 & $0 \cdot 17$ & $0.07,0.39$ \\
\hline South-east & 44 & 26,62 & 0.09 & $0.02,0.37$ & 59 & 45,72 & 0.44 & $0.18,1.09$ & 54 & 43,65 & 0.25 & $0.11,0.58$ \\
\hline South-west & 82 & 68,97 & 1 & - & 81 & 70,93 & 1 & - & 82 & 72,91 & 1 & - \\
\hline$P$ value & \multicolumn{2}{|c|}{0.0003} & \multicolumn{2}{|c|}{0.0008} & & 0001 & & $0 \cdot 0001$ & & 0001 & & 0.0001 \\
\hline Settlement & & & & & & & & & & & & \\
\hline Scattered & 72 & 52,91 & & & 74 & 59,90 & & & 73 & 61,85 & & \\
\hline Village & 53 & 35,71 & & & 54 & 45,64 & & & 54 & 46,62 & & \\
\hline Suburban & 55 & 43,66 & & & 67 & 57,77 & & & 62 & 54,70 & & \\
\hline City centre & 65 & 49,82 & & & 73 & 63,82 & & & 70 & 62,79 & & \\
\hline$P$ value & & 40 & & & & .03 & & & & .02 & & \\
\hline No. of adults in household & & & & & & & & & & & & \\
\hline 1 adult (single-parent family) & 81 & 63,99 & $5 \cdot 70$ & $1 \cdot 51,21 \cdot 54$ & 83 & 72,95 & & & 83 & 73,92 & 4.03 & $1 \cdot 71,9 \cdot 51$ \\
\hline 2 or more adults & 57 & 49,65 & 1 & - & 63 & 57,69 & & & 61 & 57,66 & 1 & - \\
\hline$P$ value & & 05 & & 0.02 & & 01 & & & & 001 & & $0 \cdot 001$ \\
\hline No. of children in household & & & & & & & & & & & & \\
\hline 1 child & 62 & 45,79 & & & 68 & 51,76 & 1 & - & 63 & 53,74 & & \\
\hline 2 children & 63 & 53,73 & & & 75 & 69,81 & $1 \cdot 44$ & $0 \cdot 72,2 \cdot 88$ & 71 & 66,76 & & \\
\hline 3 or more children & 53 & 38,67 & & & 50 & 39,61 & 0.34 & $0 \cdot 15,0.74$ & 51 & 41,60 & & \\
\hline$P$ value & & 40 & & & & 001 & & .0003 & & 003 & & \\
\hline $\begin{array}{l}\text { CC's age }(\mathrm{OR}, 95 \% \mathrm{Cl}) \\
\quad P \text { value }\end{array}$ & $1 \cdot 08$ & $\begin{array}{l}1 \cdot 02,1 \cdot 14 \\
008\end{array}$ & & & $1 \cdot 00$ & $\begin{array}{l}0 \cdot 97,1 \cdot 04 \\
\cdot 79\end{array}$ & & & $1 \cdot 03$ & $\begin{array}{l}1 \cdot 00,1 \cdot 06 \\
.06\end{array}$ & $1 \cdot 04$ & $\begin{array}{l}1 \cdot 00,1 \cdot 07 \\
0.03\end{array}$ \\
\hline CC's occupational status & & & & & & & & & & & & \\
\hline High & 74 & 51,98 & & & 77 & 60,94 & & & 76 & 62,90 & & \\
\hline Medium & 69 & 59,79 & & & 73 & 67,79 & & & 72 & 67,77 & & \\
\hline Low (including unemployed) & 66 & 47,85 & & & 51 & 36,65 & & & 56 & 45,68 & & \\
\hline Economically inactive & 30 & 16,45 & & & 47 & 32,62 & & & 41 & 30,52 & & \\
\hline$P$ value & & 007 & & & & 0007 & & & & 0001 & & \\
\hline CC's employment status & & & & & & & & & & & & \\
\hline Working & 71 & 62,80 & $4 \cdot 31$ & $2 \cdot 01,9 \cdot 25$ & 71 & 66,77 & & & 71 & 66,76 & $2 \cdot 40$ & $1 \cdot 53,3 \cdot 73$ \\
\hline Non-working & 37 & 25,50 & 1 & - & 48 & 36,60 & & & 44 & 35,53 & 1 & - \\
\hline$P$ value & & 0001 & & $0 \cdot 0001$ & & 0003 & & & & 0001 & & 0.0001 \\
\hline
\end{tabular}


the school canteen less. Considering demographic factors, households in south-western France were more likely to enrol their young children in the school canteen than those living in eastern France. In secondary-school children, the type of settlement in which the household was located was more relevant than the region: at least $90 \%$ of children from scattered rural settlements attended the school canteen $v$. $54 \%$ of children living in city centres.

When computing all these variables into a multivariateadjusted model, only some of them retained a significant $P$ value. Concerning demographic factors, school canteen attendance still varied according to region for pre-school or elementary-school children; and the effect of the settlement in which the household was located remained relevant for children attending secondary schools. The primary socio-economic factor explaining school canteen attendance was the parent's educational level, at every school level. The employment status, but not the occupational status, also influenced enrolment in school canteen, but only for children going to pre-school or upper secondary school. The single-parent status of the family led to higher school canteen attendance in pre-school children. Finally, the living standard index of the household conditioned attendance at the school canteen for secondary-school children, but this was not the case in pre-schools and elementary schools.

\section{Reasons for not attending the school canteen}

The main reason for never attending the school canteen was that somebody prepared lunch at home $(53.5$ to 59.5\%; Fig. 1). In addition, parents of children in preschool and elementary school cited the proximity between school and home $(35.0 \%$ and $38.2 \%$, respectively), the child's unwillingness to attend the canteen $(14 \cdot 4 \%$ and $7 \cdot 0 \%)$ and the price of school lunches $(7 \cdot 3 \%$ and $11 \cdot 2 \%)$. Children in lower and upper secondary schools stated their dislike of eating at the canteen $(15 \cdot 3 \%$ and $25.0 \%$, respectively), the proximity between school and home $(8.7 \%$ and $12.5 \%)$ and their preferring to eat elsewhere $(8.6 \%$ and $12 \cdot 8 \%)$. Among the children never attending the school canteen, almost $36 \%$ of them, living in rural areas or in mid-sized cities (20000 to 100000 inhabitants), did so because their house was near the school, $v$. $8 \%$ of those who lived in small cities (2000 to 20000 inhabitants; $P=0 \cdot 006$, results not shown).

\section{Discussion}

The INCA2 survey (2006-2007) was the first one to assess regular attendance at the school canteen in a nationally representative sample of children aged 3-17 years in France. Indeed, the earlier INCA survey (1998-1999) provided school canteen attendance rates of children aged 3-14 years only during the $7 \mathrm{~d}$ of the study ${ }^{(13)}$. Likewise, data from the Ministry of Education give rates of 
Table 3 Prevalence of regular and occasional school canteen (SC) attendance (\%, 95\% Cl), and multivariate-adjusted odds ratios (ORa, 95\% Cl) for SC attendance according to socioeconomic factors among children sampled attending lower and upper secondary schools in the second French national cross-sectional food consumption survey (INCA2, 2006-2007)

\begin{tabular}{|c|c|c|c|c|c|c|c|c|c|c|c|c|}
\hline & \multicolumn{4}{|c|}{ Lower secondary school } & \multicolumn{4}{|c|}{ Upper secondary school } & \multicolumn{4}{|c|}{ Total } \\
\hline & \multicolumn{2}{|c|}{ SC attendance } & \multirow{2}{*}{$\begin{array}{l}\mathrm{ORa}^{*} \\
\text { (n 507) }\end{array}$} & \multirow[b]{2}{*}{$95 \% \mathrm{Cl}$} & \multicolumn{2}{|c|}{ SC attendance } & \multirow{2}{*}{$\begin{array}{l}\mathrm{ORa}^{*} \\
(n \text { 293) }\end{array}$} & \multirow[b]{2}{*}{$95 \% \mathrm{Cl}$} & \multicolumn{2}{|c|}{ SC attendance } & \multirow{2}{*}{$\begin{array}{l}\mathrm{ORa}^{*} \\
(n \mathrm{800})\end{array}$} & \multirow[b]{2}{*}{$95 \% \mathrm{Cl}$} \\
\hline & $\%$ & $95 \% \mathrm{Cl}$ & & & $\%$ & $95 \% \mathrm{Cl}$ & & & $\%$ & $95 \% \mathrm{Cl}$ & & \\
\hline \multicolumn{13}{|l|}{ Gender } \\
\hline Boys & 75 & 68,82 & & & 69 & 56,81 & & & 73 & 67,79 & & \\
\hline Girls & 68 & 61,75 & & & 61 & 46,75 & \multirow{2}{*}{\multicolumn{6}{|c|}{$0 \cdot 12^{58,73}$}} \\
\hline$P$ value & \multicolumn{2}{|c|}{$0 \cdot 18$} & & & \multicolumn{2}{|c|}{0.38} & & & & & & \\
\hline $\begin{array}{l}\text { Child's age, OR }(95 \% \mathrm{Cl}) \\
P \text { value }\end{array}$ & \multicolumn{2}{|c|}{0.52} & & & \multicolumn{2}{|c|}{0.80} & & & \multicolumn{2}{|c|}{$0 \cdot 18$} & & \\
\hline \multicolumn{13}{|l|}{ Region } \\
\hline Ile de France & 67 & 55,79 & & & 66 & 48,81 & & & 67 & 57,77 & & \\
\hline North-west & 79 & 70,88 & & & 54 & 28,78 & & & 70 & 56,84 & & \\
\hline North-east & 61 & 49,72 & & & 57 & 39,73 & & & 59 & 49,69 & & \\
\hline South-east & 72 & 62,82 & & & 74 & 62,84 & & & 73 & 65,80 & & \\
\hline South-west & \multirow{2}{*}{\multicolumn{2}{|c|}{$0.06^{00,91}$}} & & & 86 & 71,94 & & & 81 & 71,91 & & \\
\hline$P$ value & & & & & \multicolumn{2}{|c|}{$0 \cdot 19$} & & & & 0.09 & & \\
\hline Settlement & & & & & & & & & & & & \\
\hline Scattered & 91 & 85,99 & $9 \cdot 03$ & $2 \cdot 59,31 \cdot 43$ & 93 & 84,100 & $9 \cdot 52$ & $2 \cdot 54,35 \cdot 75$ & 92 & 87,98 & $10 \cdot 29$ & $3 \cdot 49,30 \cdot 35$ \\
\hline Village & 84 & 76,92 & 3.74 & $1 \cdot 66,8 \cdot 43$ & 86 & 77,95 & 3.56 & $1 \cdot 30,9 \cdot 78$ & 84 & 78,91 & 3.55 & $1 \cdot 84,6 \cdot 84$ \\
\hline Suburban & 59 & 51,68 & $1 \cdot 04$ & $0.59,1.85$ & 59 & 45,73 & $1 \cdot 40$ & $0.62,3 \cdot 19$ & 59 & 52,67 & $1 \cdot 19$ & $0.74,1.90$ \\
\hline City centre & 59 & 47,70 & 1 & - & 48 & 27,69 & 1 & - & 54 & 43,66 & 1 & - \\
\hline$P$ value & $<0.000$ & & & $0001+$ & & 02 & & $001+$ & & $<0.0001$ & & $0001+$ \\
\hline No. of adults in household & & & & & & & & & & & & \\
\hline 1 adult (single-parent family) & 63 & 51,72 & & & 67 & 51,83 & & & 64 & 55,74 & & \\
\hline 2 or more adults & 73 & 68,78 & & & 64 & 53,75 & & & 70 & 64,75 & & \\
\hline$P$ value & & $\cdot 13$ & & & & 77 & & & & 0.32 & & \\
\hline No. of children in household & & & & & & & & & & & & \\
\hline 1 child & 68 & 61,75 & & & 73 & 64,80 & & & 70 & 65,75 & & \\
\hline 2 children & 74 & 66,81 & & & 67 & 53,79 & & & 72 & 65,79 & & \\
\hline 3 or more children & 72 & 62,82 & & & 45 & 22,71 & & & 65 & 50,77 & & \\
\hline$P$ value & & .61 & & & & 08 & & & & 0.40 & & \\
\hline $\begin{array}{l}\text { CC's age }(\mathrm{OR}, 95 \% \mathrm{Cl}) \\
P \text { value }\end{array}$ & 0.99 & $\begin{array}{l}0.95,1 \cdot 04 \\
.74\end{array}$ & & & 0.94 & $\begin{array}{l}0 \cdot 89,0 \cdot 99 \\
01\end{array}$ & & & 0.97 & $\begin{array}{l}0.94,1.01 \\
0.09\end{array}$ & & \\
\hline CC's occupational status & & & & & & & & & & & & \\
\hline High & 86 & 75,97 & & & 82 & 67,91 & & & 84 & 76, 92 & & \\
\hline Medium & 75 & 69,80 & & & 75 & 66,83 & & & 75 & 70,79 & & \\
\hline Low (including unemployed) & 65 & 51,78 & & & 54 & 34,73 & & & 62 & 51,73 & & \\
\hline Economically inactive & 61 & 24,54 & & & 33 & 15,56 & & & 51 & 36,66 & & \\
\hline$P$ value & & .06 & & & & 001 & & & & 0.0001 & & \\
\hline CC's employment status & & & & & & & & & & & & \\
\hline Working & 74 & 69,79 & & & 76 & 69,82 & $4 \cdot 10$ & $1 \cdot 87,8.95$ & 75 & 71,79 & & \\
\hline Non-working & 64 & 51,77 & & & 36 & 16,53 & 1 & - & 53 & 41,66 & & \\
\hline$P$ value & & $\cdot 13$ & & & & 0001 & & 0001 & & 0.0004 & & \\
\hline
\end{tabular}


pupils enrolled at the canteen in secondary schools, but no information on individual attendance. Additionally, the INCA2 survey provided dietary and nutritional intakes at the individual level ${ }^{(16,18)}$ that may later be compared according to the children's school canteen attendance level. Its representativeness was checked by comparison with the national census and corrected by the weighting factor applied to the children of the final sample.

However, some limitations of the INCA2 survey in studying school lunch attendance have to be emphasised. It was primarily designed to assess individual food intake and did not gather information on the schools attended by children such as size, location, presence of a canteen on the premises, catering service organisation, lunch price, etc., yet some of these characteristics were described as being associated with school lunch participation $^{(20)}$. Consequently, our work focused on the relationship between the socio-economic and demographic background of the children and their attendance at the school canteen, independently of the school characteristics. Moreover, the reasons for not participating in school lunch could not be compared between school levels, as this information was collected by a multiplechoice question and the proposals differed between age classes. Although the reasons given, other than those suggested, were probably underestimated, they were maintained as they provided qualitative information on the main barriers to school lunch attendance.

\section{School canteen attendance in 2006-2007}

In the present study, the weekly (at least once weekly) school lunch participation rate was established as from $59.0 \%$ in pre-school to $71.5 \%$ in lower secondary school, with most children using the school canteen more than three times weekly. From pre-school to lower secondary school the rate of pupils enrolled at the canteen increased and the share of occasional users decreased. In upper secondary school, school canteen attendance was slightly lower, and the share of children preferring to have lunch elsewhere increased. The higher rate of school canteen users in secondary schools may be explained by the average distance between secondary school and home, which is higher than for elementary schools. Indeed, secondary schools are fewer and more scattered than elementary schools. Therefore, more children may have to use school transportation and stay at school for lunch. This hypothesis is supported by the impact of the settlement in which the household was located on the school canteen attendance of secondary-school children.

Our results were consistent with other national data available for secondary schools, which supported the representativeness of our study. A national study carried out on public secondary schools estimated the rate of pupils enrolled in the school canteen (at least three school lunches per week) at $67 \%$ in $2005-2006^{(9)}$. Moreover, data from the Ministry of Education supplied 


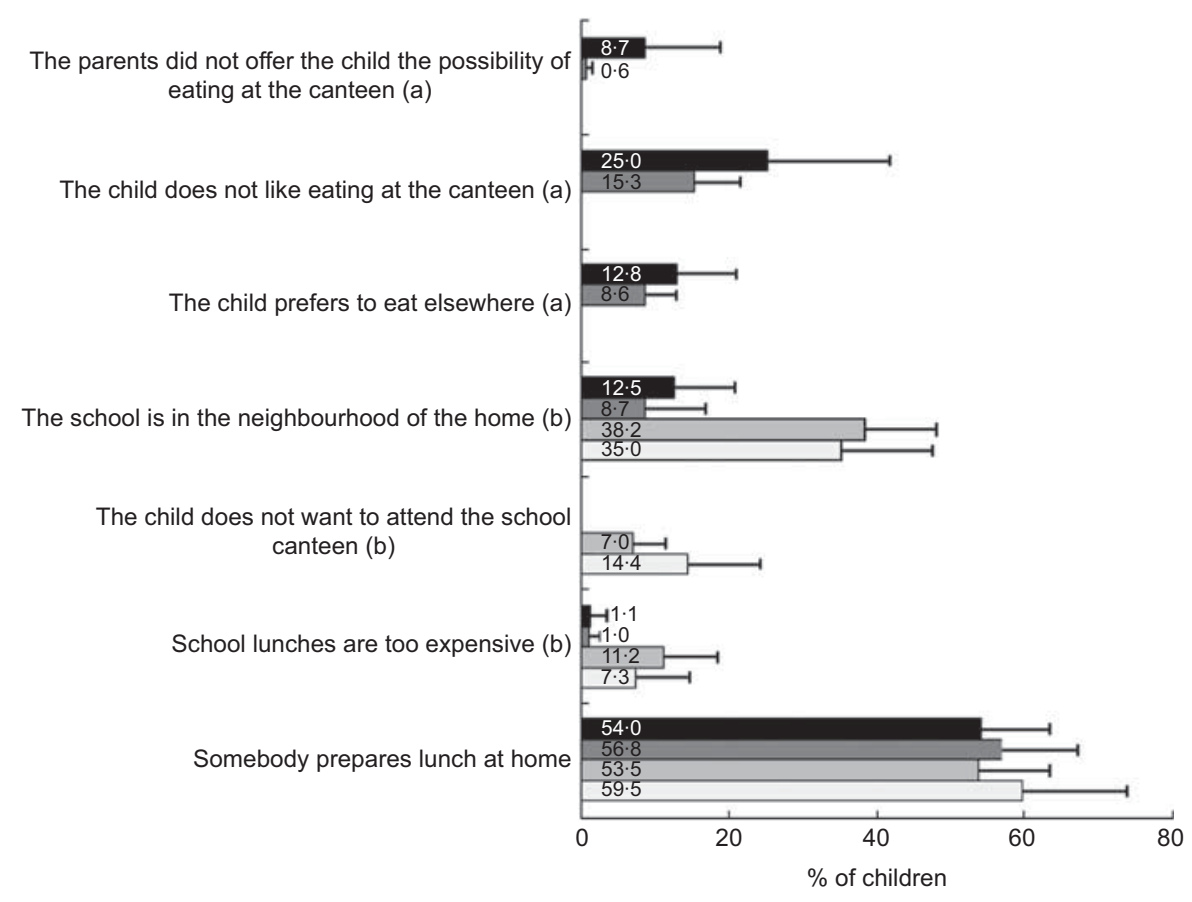

Fig. 1 Reasons for never attending the school canteen, according to school level ( $\square$, pre-school; $\square$, elementary school; $\square$, lower secondary school; $\mathbf{\square}$, upper secondary school): children aged 3-17 years sampled in the second French national cross-sectional food consumption survey (INCA2), 2006-2007. Bars represent the percentage of children reporting the answer, with 95\% confidence interval shown by error bar. (a) The answer was not suggested by default in the 3- to 10-year-olds' questionnaire, but could have been cited by the child; (b) the answer was not suggested by default in the 11- to 17-year-olds' questionnaire, but could have been cited by the child

enrolment rates of $64 \%$ in $2006-2007$, also in secondary schools ${ }^{(21)}$, including private schools. To our knowledge, no national data are available with which to compare our figures in pre-schools and elementary schools. Abroad, data on school lunch participation are also scarce and the figures mentioned were mainly lower than in France. In the USA, the last School Nutrition Dietary Assessment Study (SNDA-III) reported that 91\% of elementary- and secondary-school children ate lunch at school in 2004-2005, but the share taken in school cafeterias was not specified ${ }^{(22)}$. However, a study carried out in Pennsylvania assessed at $56.5 \%$ the average daily participation in school lunch in high schools ${ }^{(20)}$. Nearer to France, use of the school canteen was assessed at 39.3\% and 35.1\% among English children in primary and secondary schools respectively in $2008-2009^{(23)}$ and at $42 \cdot 0 \%$ of elementaryschool children in southern Spain ${ }^{(24)}$.

Compared with earlier French data, children seemed to attend the school canteen more frequently. In 1998-1999, $42 \%$ and $47 \%$ of boys and girls aged 3-14 years had at least one lunch at school during the survey week ${ }^{(13)}$. In 2006-2007, from the $7 \mathrm{~d}$ diary record, the figures were $67 \%$ and 60\%, respectively (results not shown). The increased school canteen attendance is corroborated by data from the Ministry of Education. From 1997 to 2007, school lunch enrolment increased steadily by 10 percentage points in lower secondary schools ${ }^{(21)}$. This rise corresponded with the implementation in 1997 of financial aids aimed at helping children from low-income families to have school lunch.

\section{Demographic and socio-economic variation of school lunch participation}

In 2006-2007, school canteen attendance was strongly associated with region, which is a common result in France $^{(12,13,25)}$, and with the settlement in which the household was located. The region was more discriminating in young schoolchildren and the settlement in secondaryschool children. For the latter, results concerning the reasons for not attending the school canteen suggested that the distance from home to school could easily explain the role of settlement. Children living in city centres had a higher probability of living in the vicinity of a secondary school than children living in scattered rural areas or villages. In contrast, as elementary schools are found even in scattered rural areas or villages, the effect of settlement is lower. The regional east-west gradient is more difficult to clarify. Regional socio-economic differences could be put forward but the effects of both socio-economic and demographic factors remained significant in the multivariate-adjusted regression model. Moreover, the French national study carried out on secondary public school canteens in 2005-2006 ${ }^{(9)}$ suggested that the effect of region on school lunch participation remained independently of the school 
catering characteristics and the lunch price (results not shown). Thus, it may just reflect cultural regional differences that could not be clarified further by the data available from those surveys.

School meal attendance was linked more closely with the socio-economic characteristics of the caregiver/parent (mostly the mother) than with those of the head of the household (results not shown). In the multivariateadjusted model, only the educational level of the former, but not his/her occupational status, remained associated with school canteen attendance at every school level. This result highlights the predominant role of education rather than professional occupation in school lunch participation. This hypothesis was further advanced in a study on deprived lower secondary schools in suburban Paris ${ }^{(26)}$, in which school canteen attendance was explained by the parent's relationship with the school institution. Parents who had enjoyed going to school (and thus were more likely to have a high educational level) were more inclined to enrol their child in the school canteen than those parents who had not liked school as a pupil. Nevertheless, income considerations were maintained in the multivariate model for children attending secondary schools, but not pre-schools or elementary schools. In France, the price of school lunches in pre- and elementary schools is fixed by the town council and varies automatically according to a coefficient based on household income and composition. High-income households pay the full price whereas deprived households pay a reduced price. In secondary schools, a single price is fixed by the school. Several allowances are proposed to help deprived households to afford the cost of school lunches. These allowances have to be claimed for by the families. This second system may be less effective at promoting the school canteen to low-income families because they may not claim for all the allowances they are entitled to or could encounter difficulties in completing the different application forms. This may explain why the household living standards index remained a significant factor in school canteen attendance in secondary schools only. However, the price was cited as one of the main reasons for not attending the school canteen in elementary schools. Family organisation and constraints also influenced school canteen attendance, mainly for young children. Indeed, the non-working status of the caregiver decreased the children's attendance at the school canteen, and this corresponded with the result on nonattendance because of somebody at home preparing lunch. Employment status could also explain the greater attendance of pre-school children from single-parent families: the only parent has to work and therefore should prefer that his/her young child has lunch at school.

The relationship between school lunch participation and socio-economic and geographic factors had already been described in 1999. At that time, household income was mainly advanced as the explanatory socio-economic factor $^{(13)}$. However, only the characteristics of the head of the household were available in the INCA1 survey. Our study showed that school canteen attendance was more linked to the caregiver/parent characteristics. Despite the implementation of state aids to promote the school lunch participation of children from low-income families, the socio-economic disparities persisted over the years. It has been shown that educational level and socio-economic background both play an important role in individual dietary habits ${ }^{(27-30)}$. Usually, higher socio-economic groups adopt better dietary habits according to the nutritional recommendations for a healthy diet and this seems to remain the case over the years ${ }^{(27,31)}$. These differences were often explained by a better knowledge of nutritional issues and healthy dietary behaviour in this population category ${ }^{(32,33)}$. In France, recent data from the INCA2 survey ${ }^{(34)}$ showed that consumption of the foods (fruit, vegetables, dairy products) and nutrients (sodium, calcium) targeted by school meal recommendations ${ }^{(35)}$ were socio-economically dependent and that children from lower education backgrounds were less in line with the recommendations. Moreover, the INCA2 survey also pointed out a strong inverse relationship between parents' educational level and child overweight ${ }^{(19)}$. In Great Britain, a similar educational gradient was mentioned in the nutritional intake of pre-school children ${ }^{(28)}$. From a public health point of view, the low school lunch participation of children from deprived backgrounds could decrease the effectiveness of the upcoming law on the nutritional composition of school meals that is designed to encourage them to adopt healthier dietary habits. Implementation of supporting policy on school meal prices may counteract this effect, like in the USA where school lunch programme participation increased positively with the percentage of students eligible for free/reduced-price meals ${ }^{(20)}$. Furthermore, these children could then be a vector of healthier dietary habits into their family ${ }^{(36,37)}$. Otherwise, the nutritional policy on school lunches may increase existing differences in dietary behaviour among children according to their socioeconomic background ${ }^{(38)}$.

\section{Conclusions}

In France, school lunch participation is high, particularly in secondary schools. Like dietary habits, overweight and many other health issues, school canteen attendance is associated with socio-economic and demographic factors, mainly the educational level of the caregiver/parent. In a context of future regulations concerning school meal composition, ways to improve the participation of children from low socio-economic backgrounds, such as increasing financial aids, should be found in order to lead these children towards healthier dietary habits on a longterm basis. 


\section{Acknowledgements}

INCA2 was supported by a grant from the French Food Safety Agency (AFSSA). There were no conflicts of interest in the preparation of the paper. C.D. analysed the data and wrote the paper. L.L., S.L., J.-L.V. and D.T. helped to write the paper. G.C.-T., C.D., A.D., L.L., S.L. and J.-L.V. contributed to the design and data collection of the INCA2 survey. All of the authors reviewed the manuscript. The authors thank the ISL team (Institut de Sondage Lavialle) for the collection of data, and all the families for their cooperation.

\section{References}

1. Jaime PC \& Lock K (2009) Do school based food and nutrition policies improve diet and reduce obesity? Prev Med 48, 45-53.

2. Vereecken CA, Bobelijn K \& Maes L (2005) School food policy at primary and secondary schools in BelgiumFlanders: does it influence young people's food habits? Eur J Clin Nutr 59, 271-277.

3. Food Standards Agency (2004) School Meals in Secondary Schools in England. Research Report RR557. http:// www.dfes.gov.uk/research/data/uploadfiles/RR557.pdf (accessed November 2009).

4. Cullen KW, Watson K, Zakeri I et al. (2006) Exploring changes in middle-school student lunch consumption after local school food service policy modifications. Public Health Nutr 9, 814-820.

5. Briefel RR, Crepinsek MK, Cabili C et al. (2009) School food environments and practices affect dietary behaviors of US public school children. J Am Diet Assoc 109, 2 Suppl., S91-S107.

6. Hercberg S, Chat-Yung S \& Chauliac M (2008) The French National Nutrition and Health Program: 2001-2006-2010. Int J Public Health 53, 68-77.

7. Anon. (2004) Collation matinale à l'école. Note de service n²004-095 du 25 mars 2004. DESCO, Ministère de l'Education nationale. http://www.sante.gouv.fr/pointsur/ nutrition/pol_nutri422.htm (accessed July 2010).

8. Anon. (2004) Loi n'2004-806 du 9 août 2004 relative à la politique de santé publique. Journal Officiel de la République Française, p. 14277. http://www.legifrance.gouv.fr/ jopdf/common/jo_pdf.jsp?numJO $=0 \&$ datejO $=20040811 \&$ num Texte $=4$ \&pageDebut $=14277$ \&pageFin $=14337 \quad$ (accessed November 2009).

9. Dubuisson C, Lioret S, Calamassi-Tran G et al. (2009) School meals in French secondary state schools with regard to the national recommendations. Br J Nutr 102, 293-301.

10. Anon. (2001) Circulaire $\mathrm{n}^{\circ} 2001-118$ du 25 juin 2001. Composition des repas servis en restauration scolaire et sécurité des aliments. Bulletin officiel du ministère de l'Education nationale et du ministère de la Recherche. http://www.education.gouv.fr/bo/2001/special9/som.htm (accessed November 2009).

11. Czernichow S\& Martin A (2000) Nutrition and school catering, from school garden to high school: review. http:// www.afssa.fr/Documents/PASER-Ra-Restoscolaire2000.pdf (accessed November 2009).

12. Floch J-M (1999) La fréquentation de la restauration scolaire dans les collèges publics. Note d'information $d u$ Ministère de l'éducation nationale 99, 23.

13. Lafay L, Volatier J-L \& Martin A (2002) La restauration scolaire dans l'enquête INCA - Associations entre facteurs socio-démographiques, mode de vie et fréquentation de la restauration scolaire (1ère partie). Cah Nutr Diet 37, 36-44.
14. Lioret S, Maire B, Volatier JL et al. (2007) Child overweight in France and its relationship with physical activity, sedentary behaviour and socioeconomic status. Eur J Clin Nutr 61, 509-516.

15. Lioret S, Touvier M, Lafay L et al. (2008) Dietary and physical activity patterns in French children are related to overweight and socioeconomic status. J Nutr 138, 101-107.

16. Lioret S, Dubuisson C, Dufour A et al. (2010) Trends in food intake in French children from 1999 to 2007: results from the INCA (etude Individuelle Nationale des Consommations Alimentaires) dietary surveys. Br J Nutr 103, 585-601.

17. Dufour A, Lafay L \& Volatier J-L (2008) La mesure des consommations alimentaires par l'enquête INCA2. In Méthodes de sondage, pp. 132-138. Paris: Dunod.

18. Dubuisson C, Lioret S, Touvier M et al. (2010) Trends in food and nutritional intakes of French adults from 1999 to 2007: results from the INCA surveys. Br J Nutr 103, $1035-1048$.

19. Lioret S, Touvier M, Dubuisson C et al. (2009) Trends in child overweight rates and energy intake in France from 1999 to 2007: relationships with socioeconomic status. Obesity (Silver Spring) 17, 1092-1100.

20. Probart C, McDonnell E, Hartman T et al. (2006) Factors associated with the offering and sale of competitive foods and school lunch participation. J Am Diet Assoc 106, 242-247.

21. Direction de l'évaluation, de la prospective et de la performance (2007) Répères et références statistiques sur les enseignements, la formation et la recherche, Septembre 2007. http://www.education.gouv.fr/cid5489/reperes-etreferences-statistiques.html (accessed November 2009).

22. Briefel RR, Wilson A \& Gleason PM (2009) Consumption of low-nutrient, energy-dense foods and beverages at school, home, and other locations among school lunch participants and nonparticipants. J Am Diet Assoc 109, 2 Suppl., S79-S90.

23. Nelson M, Nicholas J, Wood L et al. (2009) Take up of school lunches in England in 2008-2009. http://www. schoolfoodtrust.org.uk/UploadDocs/Library/Documents/ni52_ statistical_release08-09.pdf (accessed November 2009).

24. Lopez-Frias M, Nestares T, Ianez I et al. (2005) Nutrient intake adequacy in schoolchildren from a Mediterranean area (southern Spain). Influence of the use of the school canteen. Int J Vitam Nutr Res 75, 312-319.

25. AFSSA (2007) Evaluation de la connaissance et de l'application de la circulaire du 25 juin 2001 relative à la composition des repas servis et à la sécurité des aliments dans les établissements publics du second degré (2005-2006). http://www.afssa.fr/Documents/PASER-RaRestoScol2007.pdf (accessed May 2010).

26. César C (2006) La restauration scolaire au collège: c'est bon pour qui? La lettre scientifique de l'IFN issue 111.

27. Kant AK \& Graubard BI (2007) Secular trends in the association of socio-economic position with self-reported dietary attributes and biomarkers in the US population: National Health and Nutrition Examination Survey (NHANES) 1971-1975 to NHANES 1999-2002. Public Health Nutr 10, 158-167.

28. Watt RG, Dykes J \& Sheiham A (2001) Socio-economic determinants of selected dietary indicators in British preschool children. Public Health Nutr 4, 1229-1233.

29. Perrin AE, Dallongeville J, Ducimetiere P et al. (2005) Interactions between traditional regional determinants and socio-economic status on dietary patterns in a sample of French men. Br J Nutr 93, 109-114.

30. Giskes K, Turrell G, van Lenthe FJ et al. (2006) A multilevel study of socio-economic inequalities in food choice behaviour and dietary intake among the Dutch population: the GLOBE study. Public Health Nutr 9, 75-83. 
31. Hulshof KF, Brussaard JH, Kruizinga AG et al. (2003) Socioeconomic status, dietary intake and 10 y trends: the Dutch National Food Consumption Survey. Eur J Clin Nutr 57, 128-137.

32. Coveney J (2005) A qualitative study exploring socioeconomic differences in parental lay knowledge of food and health: implications for public health nutrition. Public Health Nutr 8, 290-297.

33. Beydoun MA \& Wang Y (2008) Do nutrition knowledge and beliefs modify the association of socio-economic factors and diet quality among US adults? Prev Med 46, 145-153.

34. AFSSA (2009) Summary of the report of the 2006/2007 Individual and National Survey on Food Consumption 2 (INCA 2). http://www.afssa.fr/cgi-bin/countdocs.cgi? Documents/PASER-Sy-INCA2.pdf (accessed November 2009).
35. Groupe d'étude des marchés de restauration collective et de nutrition (2007) Recommandation relative à la nutrition du 4 mai 2007. http://www.minefe.gouv.fr/directions_ services/daj/guide/gpem/nutrition/nutrition.pdf (accessed November 2009).

36. Borys JM \& Lafay L (2000) L'information nutritionnelle des enfants pour modifier les habitudes alimentaires de toute la famille. Rev Med Suisse Romande 120, 207-209.

37. Basdevant A, Boute D \& Borys JM (1999) Who should be educated? Education strategies: could children educate their parents? Int J Obes Relat Metab Disord 23, Suppl. 4, S10-S12.

38. Bere E, Veierod MB \& Klepp KI (2005) The Norwegian School Fruit Programme: evaluating paid vs. no-cost subscriptions. Prev Med 41, 463-470. 